ARTíCUlo

\title{
Estudio comparativo de escamas de las líneas laterales y corporales del Bacalao de profundidad Dissostichus eleginoides (Teleostei: Nototheniidae)
}

Comparative study of lateral lines and body scales of Patagonian toothfish Dissostichus eleginoides (Teleostei: Nototheniidae)

\section{Sylvia Sáez ${ }^{*}$ y Roberto Jaramillo ${ }^{1}$}

\author{
${ }^{1}$ Instituto de Ciencias Marinas y Limnológicas, Universidad Austral de Chile, Casilla 567, Valdivia, Chile \\ *Autor corresponsal: sylvia.saez@uach.cl
}

\begin{abstract}
The morphology of the scales of both lateral lines of $D$. eleginoides was studied and compared with body scales obtained from twelve different areas along the antero-posterior and dorso-ventral axes of trunk. The scales of both lateral lines belong to the cycloid and oval types and consist of a scale plate, a central tube with an anterior opening (which is absent in some scales of the middle part of the trunk) and a posterior opening. The morphological analysis revealed no differences between the scales located at both lateral lines, while the body scales differ from the lateral line scales by absence of a central tube. Most of the scales of the anterior sector of the body are cycloid and some ctenoid. In contrast, the middle and posterior areas at both sides of the body bear both types of scales. The results obtained provide new basic morphological evidence for the presence of scales in both lateral lines and body scales. Further histological studies of the scales of both lateral lines are needed to determine the neural structural connection between these lines in order to achieve a better understanding of the characteristics of the mechanosensory system of $D$. eleginoides.
\end{abstract}

\begin{abstract}
Key words: Lepidology, lateral line, morphology, Chile
Resumen.- Se estudió la morfología de las escamas situadas en ambas líneas laterales de D. eleginoides y fueron comparadas con las escamas corporales obtenidas de 12 sectores diferentes a lo largo de los ejes antero-posterior y dorso-ventral del tronco. Las escamas de ambas líneas son del tipo cicloídeas y ovaladas; están constituidas por una placa de la escama, un tubo central con una abertura anterior (ausente en algunas escamas del sector medio del tronco) y otra posterior. El análisis morfológico no mostró diferencias entre las escamas de ambas líneas; sin embargo, las escamas corporales se diferencian de las escamas de la línea lateral por la ausencia del tubo central. La mayoría de las escamas del sector anterior del cuerpo son cicloídeas y solo algunas son del tipo ctenoídeas; mientras que en la zona media y posterior es característica la presencia de ambos tipos de escamas en el lado derecho e izquierdo del cuerpo. Los resultados obtenidos entregan nuevos antecedentes morfológicos básicos tanto para las escamas presentes en ambas líneas laterales como las corporales. Es recomendable en el caso de las escamas de las líneas laterales continuar con estudios histológicos para determinar la conexión nerviosa entre ambas y obtener así una visión más completa del sistema mecanosensorial de $D$. eleginoides.
\end{abstract}

Palabras clave: Lepidología, línea lateral, morfología, Chile

\section{INTRODUCCIÓN}

Las escamas son placas osificadas que presentan una amplia diversidad morfológica con un rol hidrodinámico y de protección para el pez (Zhu et al. 2012, Wainwright \& Lauder 2016). El estudio de la morfología y el patrón de escamación (lepidología) han sido herramientas ampliamente usadas en la diagnosis de especies, sistemática y análisis filogenético de peces (Batts 1964, Lippitisch 1990, Roberts 1993, Wonsettler \& Webb 1997, Jawad 2005, Voronina 2007, Alkaladi et al. 2013, Ansari et al. 2016, Teimori 2016, Voronina \& Hughes 2011, 2017).
La escama de la línea lateral, a menudo llamada escama tubular o escama porosa, situada sobre el tronco del pez, se caracteriza por presentar una placa de la escama y un tubo (también denominado canal en voladizo, bóveda ósea o cubierta de canal) que es la osificación de la pared del tejido conectivo del canal del tronco (Voronina \& Hughes 2017). Dicho tubo presenta una abertura anterior o rostral y otra posterior o caudal (Jawad 2005, Voronina \& Hughes 2011, 2017). 
La escama de la línea lateral es una estructura compuesta que resulta del desarrollo interconectado de la escama común y el canal del tronco (Webb 1989, Wonsettler \& Webb 1997, Voronina \& Hughes 2011, 2017). El canal del tronco es un canal continuo que se forma por la superposición de las escamas en la línea lateral y está perforado regularmente por poros epiteliales, que vinculan el lumen del canal con el ambiente externo (Wonsettler \& Webb 1997).

La línea lateral está compuesta por mecanorreceptores, llamados neuromastos, unidad funcional del sistema que se distribuye sobre la cabeza, tronco y aleta caudal del pez (Gompel et al. 2001, Wada et al. 2008, Ghysen et al. 2010). Estos neuromastos pueden ser de dos tipos: los neuromastos superficiales que se encuentran libremente en la superficie del cuerpo, y cuyas cúpulas son bañadas por el agua, y los neuromastos de canal, situados al interior de canales óseos, que poseen poros, por medio de los cuales se comunican con el exterior (Webb 1989, 2014; Kasumyan 2003, Barbin \& Humphrey 2009).

La escama modificada de la línea lateral es capaz de detectar cambios o disturbios en el agua próxima al pez y provoca variaciones de presión a lo largo de la línea lateral haciendo que el estímulo ingrese al poro del tubo de la escama y luego al neuromasto de canal, el cual recibe la información que es captada por la cúpula y comunicada a los estereocilios, siendo el kinocilio el que transmite la información por medio de neuronas aferentes hacia el cerebro (Modgans et al. 2003, Webb 1989, 2014).

Las estructuras que forman la escama de la línea lateral de los peces pueden variar según los taxa (Voronina \& Hughes 2017); dicha variación por lo general se concentra en 3 caracteres de la escama: el grado de modificación de la placa, la posición de la abertura posterior y la integridad del tubo (Webb 2014, Voronina \& Hughes 2011, 2017).

En aquellas especies con más de una línea lateral, como es el caso de los nototénidos Dissostichus mawsoni Norman, 1937 y D. eleginoides (Smitt, 1898), que presentan una línea lateral disyunta -donde la parte anterior y posterior de la línea están separadas por un espacio de una o más hileras de escamas-, también es posible observar diferencias (Voronina \& Hughes 2017). Del mismo modo, en especies con 3 líneas laterales, se ha descrito diferencias entre las escamas, como en el caso de Pionodraco evansii (Regan, 1914) de la familia Bathydraconidae (Voronina \& Hughes 2017).
A pesar que las escamas que forman las dos líneas laterales de $D$. eleginoides, son parte importante del sistema mecanosensorial de la especie, éstas han sido escasamente estudiadas y por tanto se desconoce la morfología de las escamas de ambas líneas. Al respecto, se puede citar como antecedente único, la descripción de Voronina \& Hughes (2017) quienes describen las escamas de D. eleginoides como tubulares modificadas, sin detallar el sector de la línea lateral desde donde fueron obtenidas y tampoco indican a partir de cual línea del tronco provienen.

Por lo expuesto anteriormente y considerando la escasa información acerca de las características morfológicas de las escamas que forman las dos líneas laterales de $D$. eleginoides, se realizó un estudio lepidológico en ejemplares adultos para determinar la forma y disposición de estas escamas con el fin de identificar y caracterizar dichas estructuras y establecer posibles diferencias y/o similitudes entre las dos líneas laterales del tronco. Además, se estudiaron las escamas corporales situadas en las cercanías de ambas líneas, con el fin de realizar una comparación morfológica de ambos tipos de escamas, obteniendo así un registro completo de la conformación lepidológica del tronco de D. eleginoides.

\section{MATERIALES Y MÉTODOS}

Para el estudio de las escamas de la línea lateral del tronco (LLT) de D. eleginoides, se utilizaron dos ejemplares de longitud total de 600 y $610 \mathrm{~mm}$ provenientes de capturas realizadas a 15 millas náuticas de la ciudad de Valdivia (3948'30"S y 7314'30”O), sur de Chile.

Se obtuvo un total de 228 escamas, 25 de las cuales provinieron de la línea lateral superior (LLS) y 45 de la línea lateral media (LLM), las 158 escamas restantes fueron del tipo corporal (Fig. 1A). Las escamas de LLT de ambos lados del cuerpo fueron extraídas bajo un estereomicroscopio Leica $^{\circledR}$ EZ4D con la ayuda de pinzas. En la LLS, se consideraron dos sectores: anterior y medio, mientras que en la LLM los sectores fueron: medio y posterior (Fig. 1A) (Lloris \& Rucabado 1991).

Una vez extraídas, las escamas fueron lavadas en agua destilada, para luego ser teñidas en una solución de alizarina al 1\% durante $15 \mathrm{~min}$; secadas con papel absorbente y finalmente, montadas en un portaobjeto para su observación. 

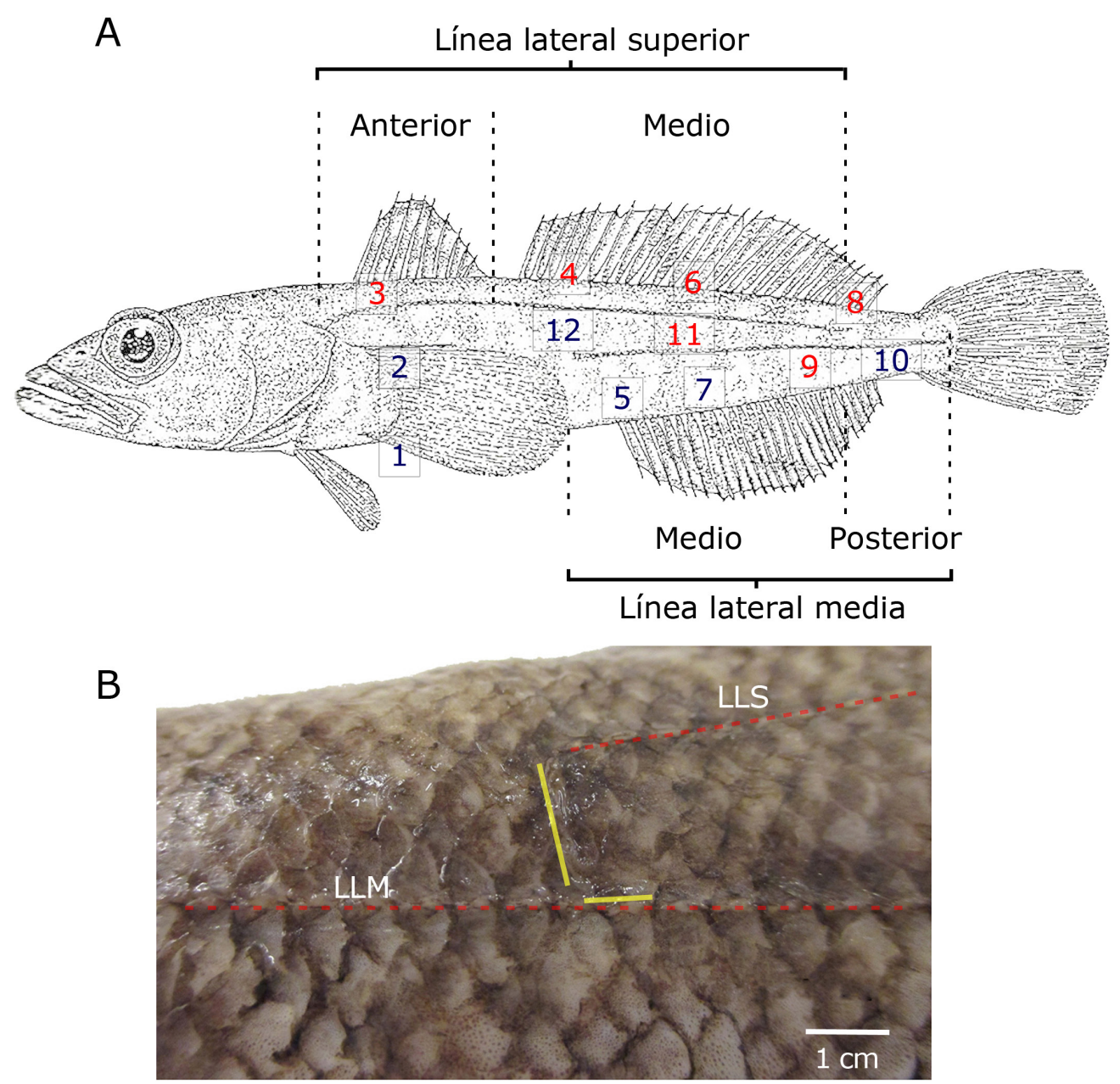

Dissostichus eleginoides

Figura 1. A) Esquema que muestra las áreas del cuerpo (12 sectores) y puntos de extracción de escamas corporales en $D$. eleginoides, los números en rojo indican la presencia de escamas ctenoídeas y los números en azul indican escamas cicloídeas (modificado de Lloris \& Rucabado 1991), B) línea amarilla muestra segmento perpendicular sin escamas entre la línea lateral superior (LLS) y la línea lateral media (LLM) del lado derecho del cuerpo / A) Diagram showing body areas (12 sectors) and body scale extraction point in D. eleginoides; red numbers indicates the presence of ctenoid scales and blue numbers indicate presence of cycloid scales (modified from Lloris \& Rucabado 1991), B) yellow line shows scaleless perpendicular segment between upper lateral line (LLS) and middle lateral line (LLM) on right side of body

Las escamas corporales (EC) se obtuvieron a partir de 12 sectores diferentes a lo largo de los ejes antero-posterior y dorso-ventral del cuerpo (Fig. 1A). Luego de extraer las escamas, fueron lavadas con agua destilada y conservadas en alcohol al 50\%. Todas las escamas (LLS, LLM y EC) fueron observadas y fotografiadas en un estereomicroscopio Leica ${ }^{\circledR}$ CME. Los detalles finos de las escamas fueron fotografiados en un microscopio Olympus ${ }^{\circledR} \mathrm{CX} 31$ con cámara integrada.

Cada escama fue analizada morfológicamente, siguiendo la nomenclatura propuesta por Jawad (2005), Voronina \& Hughes (2017) y Teimori (2016).

\section{Resultados}

D. eleginoides presenta dos líneas laterales en el tronco compuestas por escamas modificadas: la línea lateral superior (LLS) y una línea lateral media (LLM) (Fig. 1A). La LLS se inicia en el extremo superior del opérculo y finaliza por debajo del término de la segunda aleta dorsal. Como se muestra en la Figura 1B, a continuación de la LLS hay un pequeño segmento sin escamas en forma de L que baja desde el extremo distal de la LLS de manera perpendicular a la LLM y finaliza su trayecto sobre dos escamas tubulares de esta línea. Dicho segmento fue observado en ambos lados del cuerpo y en cada uno de los dos ejemplares estudiados. Por su parte, la LLM se origina en las cercanías del borde posterior de la aleta pectoral y se extiende hasta el pedúnculo caudal (Fig. 1A). 
Todas las escamas son del tipo modificadas tubular, donde el campo posterior de la placa de la escama se ha modificado y está involucrado en la formación de la abertura posterior del tubo.

Las principales características morfológicas de las escamas estudiadas son:

1) Escamas de la línea lateral superior y media:

Las escamas de la línea lateral superior (LLS) y media (LLM) de D. eleginoides, son del tipo cicloídeas y ovaladas; están formadas por un campo anterior, un campo posterior, dos campos laterales, un tubo central (habitualmente con dos aberturas, una anterior y otra posterior) y una placa de la escama (Fig. 2A).

El campo anterior (o rostral), es el sector que permanece embebido en la epidermis, donde se encuentran los radii alternándose con los circuli interradiales (Fig. 2B). Los contornos del campo anterior varían desde suaves a irregulares (independientemente del sector del tronco), mientras que los contornos de los campos laterales varían desde rectos a levemente sinuosos.
El campo posterior (o caudal) es el sector expuesto, donde se observan principalmente los circuli (más oscuros), alternándose con los annuli (más claros). En este campo se localiza la abertura posterior del tubo central (Fig. 2C).

Las escamas del sector anterior y medio de la LLS, tienen un tubo central que posee mayoritariamente ambas aberturas (Fig. 2D). Sin embargo, en algunos sectores es posible encontrar escamas sin abertura anterior (Fig. 2E). Es así como del total de 14 escamas extraídas del sector medio del tronco del lado derecho del cuerpo, solo una escama no presentó abertura anterior; del mismo modo, solo una escama (de un total de 13 escamas extraídas) del sector anterior del tronco del lado izquierdo no presentó abertura anterior.

En las escamas de la LLM se conservan las variaciones morfológicas descritas para las escamas de la LLS. En este caso, 8 de las 21 escamas observadas provenientes del sector medio del lado derecho del cuerpo no tenían abertura anterior mientras que 9 de las 30 escamas provenientes de los sectores medio y posterior de toda la LLM del izquierdo, no presentaban abertura anterior.
A

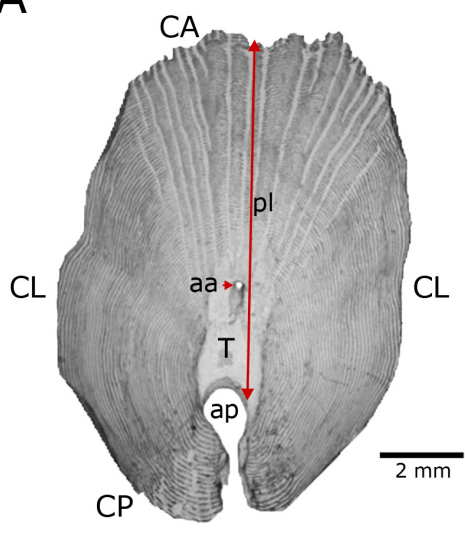

D
B

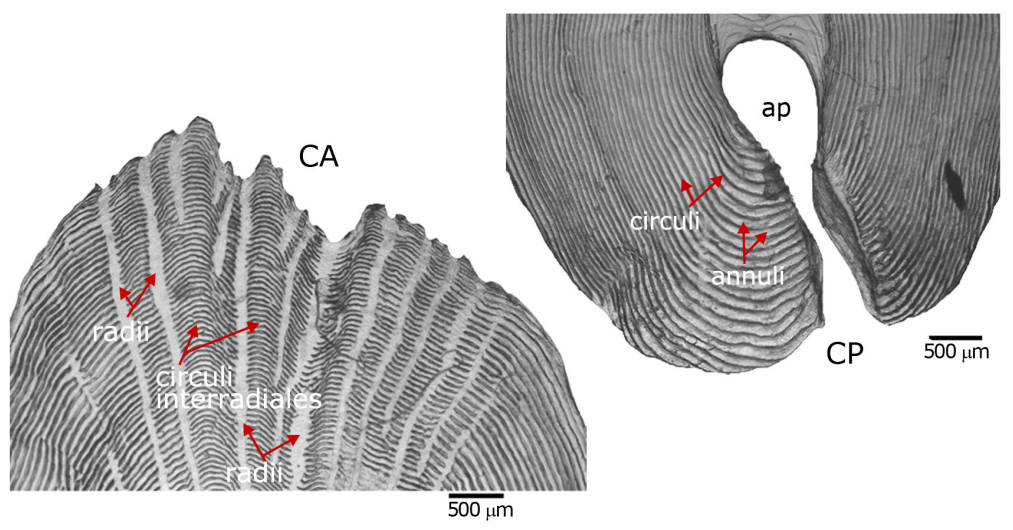

E
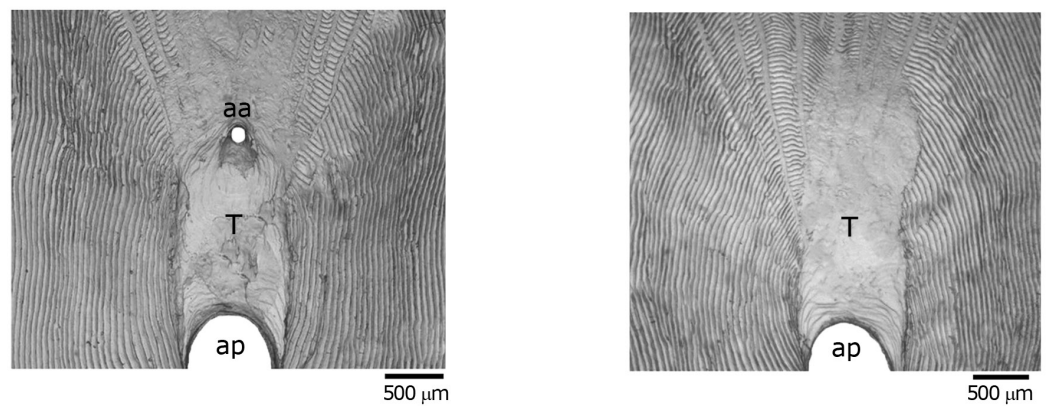

Figura 2. A) Vista general de una escama de la línea lateral: CA campo anterior, CP campo posterior, CL campo lateral, aa abertura anterior, ap abertura posterior, $\mathrm{T}$ tubo central, $\mathrm{pl}$ placa de la escama, B) Campo anterior de una escama de la línea lateral superior derecha (4X), C) Campo posterior de una escama de la línea lateral derecha (4X), D) Escama de la línea lateral media derecha del cuerpo con abertura anterior y posterior del tubo (4X), E) Escama de la línea lateral superior derecha sin abertura anterior (4X) / A) General view of a lateral line scale: CA anterior field, CP posterior field, $\mathrm{CL}$ lateral field, aa anterior opening, ap posterior opening, T central tube, pl scale plate, B) Anterior field of a scale of the upper right lateral line (4X), C) Posterior field of a scale of the right lateral line (4X), D) Scale of the right middle lateral line of the body with anterior and posterior opening of the tube (4X), E) Scale of the right upper lateral line without anterior opening (4X) 


\section{2) Escamas corporales:}

Estas se caracterizan por su forma casi ovalada además de presentar un campo anterior (o rostral), un campo posterior (o caudal) y dos campos laterales y se diferencian de aquellas de las líneas laterales por la ausencia del tubo central. En ellas se aprecian los circuli, annuli, radii y un focus parte central de la escama (Fig. 3A, B, C). El campo anterior es el sector que permanece embebido en la epidermis, es redondeado y con numerosos radii (Fig. 3B). El campo posterior es el sector expuesto (y con melanóforos) y más angosto de la escama (Fig. 3C). En este sector se observan los circuli y annuli, y además ctenos cuyo número varía de unos pocos a una veintena (Fig. 4A, 4B). Los campos laterales son redondeados (Fig. 3A).

De los 12 sectores analizados (Fig. 1A), solo se observó diferencias entre las escamas del sector anterior del cuerpo (sector 3), donde la mayoría de ellas son cicloídeas (Fig. 3A, 3B, 3C) y algunas ctenoídeas. En la zona media y posterior (sectores 4, 6, 8, 9 y 11) hay una mezcla entre escamas cicloídeas y ctenoídeas (Fig. 4A, 4B). Ambos tipos de escamas se presentan tanto en el lado derecho como izquierdo del cuerpo.

\section{Discusión}

La literatura indica que las escamas de la línea lateral forman canales tubulares que se encuentran dentro de una serie horizontal de escamas superpuestas, y que en la mayoría de los peces se disponen en una sola línea de escamas que comienza en el margen caudal del cráneo y se extiende hasta el pedúnculo caudal, formando el canal del tronco (Wonsettler \& Webb 1997, Webb \& Ramsay 2017). Los resultados obtenidos sugieren que $D$. eleginoides no sigue esta generalidad, puesto que en este caso es la segunda línea (LLM), la que se extiende hasta el pedúnculo caudal. Por su parte, las escamas de ambas líneas laterales conservan características morfológicas similares en todos los sectores estudiados y coinciden con el tipo de escamas definido como tubular modificada descrita por Voronina \& Hughes (2017).
A

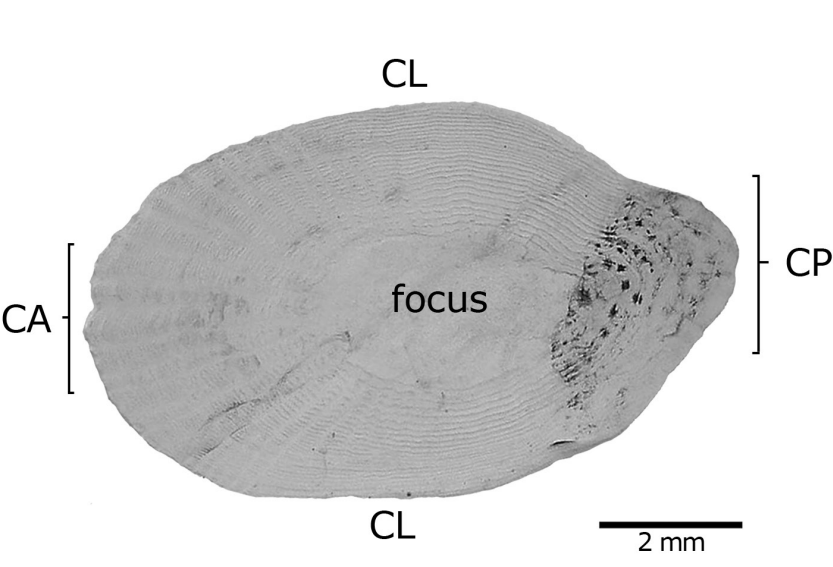

B

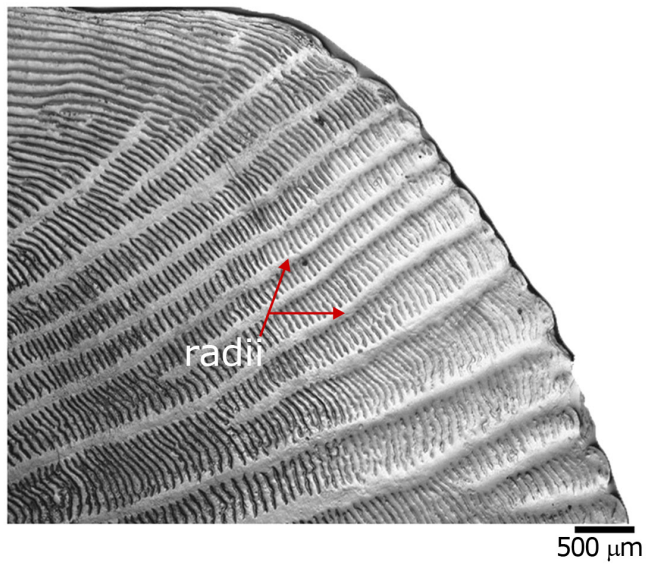

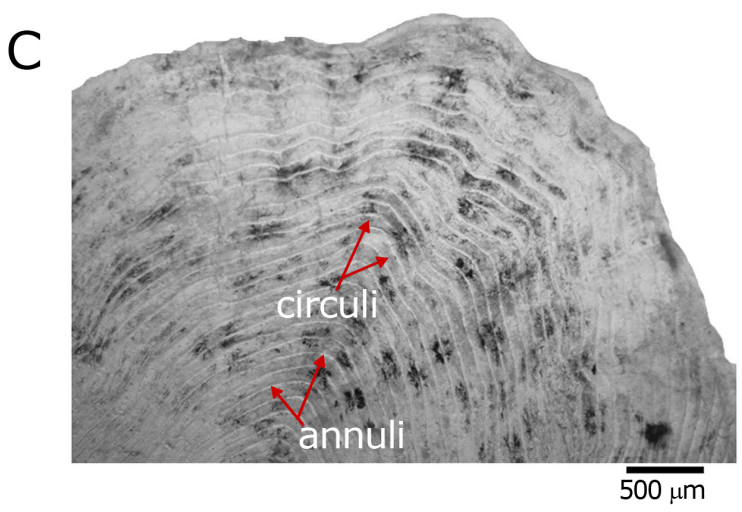

Figura 3. A) Partes de una escama corporal, CA campo anterior, CP campo posterior, CL campo lateral, B) Campo anterior de una escama corporal del sector medio (sector 9) (4X), C) Campo posterior de una escama corporal sin ctenos del sector medio (sector 5) (4X) / A) Sections of a body scale, CA anterior field, CP posterior field, CL lateral field, B) Anterior field of a middle sector body scale (section 9) (4X), C) Posterior field of a body scale without ctenii from the middle sector (section 5) (4X) 
A

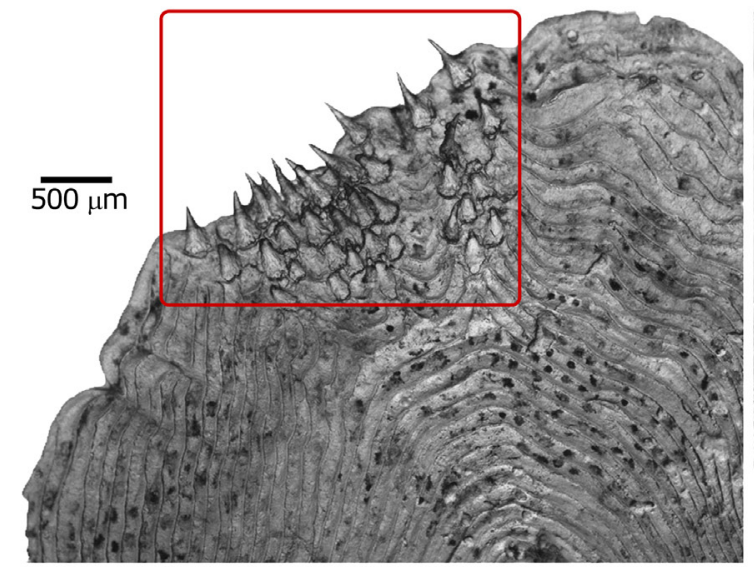

B

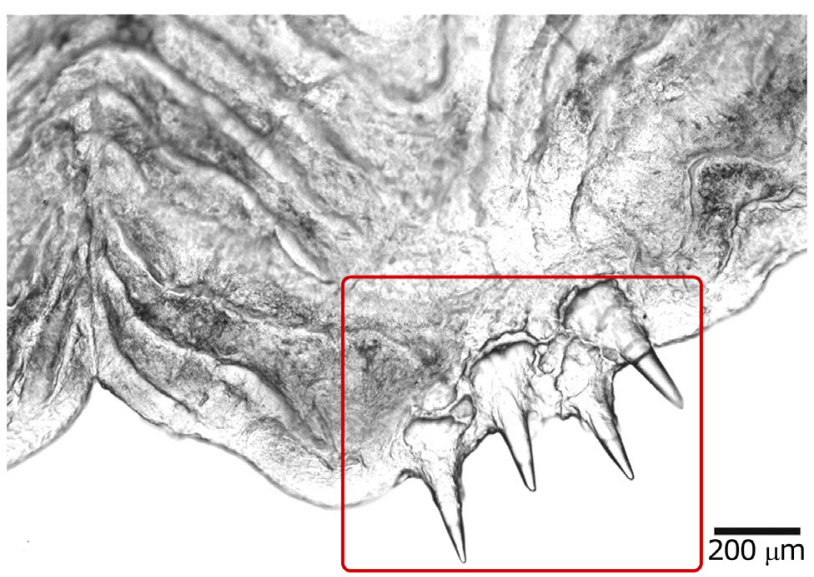

Figura 4. Campo posterior de una escama corporal con ctenos (recuadro rojo). A) Escama del sector medio de la línea lateral media (sector 9) (4X), B) Escama del sector medio de la línea lateral superior (sector 6) (10 X) / Posterior field of a body scale with ctenii (red box). A) Scale of middle sector of the middle lateral line (section 9) $(4 \mathrm{X})$, B) Scale of middle sector of the upper lateral line (section 6) (10X)

Otro aspecto revelado en este estudio es la existencia de un pequeño segmento perpendicular que se extiende entre la LLS y LLM. Es posible que este segmento corresponda a la continuación de la LLS que se extiende hasta el borde de la LLM. Sin embargo, se hace necesario realizar estudios histológicos que permitan determinar si existe algún tipo de neuromasto (en canal o superficiales) y/o alguna similitud histológica con la LLS. Esta propuesta se debe a la evidencia aportada por Wonsettler \& Webb (1997) quienes al realizar un análisis histológico de dos especies de escorpénidos (Hexagrammos decagrammus y $H$. stelleri), que poseen 5 líneas laterales cada uno, revelaron que solo una línea lateral (o canal) presentaba receptores mecanosensoriales y que por lo tanto las 5 líneas no eran equivalentes funcionalmente.

Al considerar los antecedentes previamente citados es posible asumir que la presencia de dos líneas laterales en D. eleginoides, no necesariamente implica una duplicidad de estructuras y que probablemente solo una de ellas tenga la función mecanosensorial, por tal razón sería de suma importancia realizar, además, un análisis histológico de ambas líneas laterales con el fin de revelar la presencia/ ausencia de neuromastos.

Webb \& Ramsay (2017) señalan que las escamas de la línea lateral forman una serie superpuesta en la que el campo posterior expuesto de una escama se dispone sobre el campo anterior de la siguiente. Los resultados de este trabajo indican que algunas escamas del sector medio, en especial en la LLM de ambos lados del tronco $D$. eleginoides se caracterizan por la ausencia de la abertura anterior. Esto sugiere que las escamas que presentan ambas aberturas se intercalan con aquellas sin una abertura anterior, hecho que no alteraría la funcionalidad de las escamas, ya que al estar superpuestas unas con otras, la corriente circulatoria creada en la escama con ambas aberturas traspasaría la información a la escama con abertura posterior, permitiendo con ello percibir la información hidrodinámica necesaria para que el pez determine la dirección, velocidad, tamaño y forma de los objetos dispuestos a su alrededor (Vogel \& Bleckmann 2000, Modgans et al. 2003, Ishida et al. 2014, Ristroph et al. 2015).

En relación con las escamas corporales, este estudio reveló la presencia de escamas cicloideas y ctenoídeas, desde el sector anterior del cuerpo hacia el posterior, hecho que desde el punto de vista hidrodinámico pudiera indicar la ocurrencia de una transición desde una capa límite laminar a una turbulenta (Wainwright \& Lauder 2016). De esta manera, las escamas cicloideas del sector anterior del cuerpo reducirían la resistencia al paso del agua creando una capa de borde laminar al presentar una superficie lisa (Burdak 1986). Por otra parte, como las escamas se encuentran en contacto con la epidermis y el mucus, podrían disminuir la fricción entre las que se superponen facilitando así su deslizamiento, permitiendo con ello una mayor curvatura corporal antes que las escamas empiecen a doblarse y ejerzan una resistencia a esta curvatura. En este aspecto, no es extraño encontrar ambos tipos de escamas sobre el cuerpo de otros Perciformes como, por ejemplo, en las familias Percidae, Centrarchidae y Tripterygiidae (Roberts 1993, Jawad 2005).

Todas las estructuras que forman parte de una escama, sean estas de la línea lateral o corporal, (circuli, radii y ctenos así como la forma de los bordes de las escamas) tienen propiedades mecánicas e hidrodinámicas (Jawad 2005, Wainwright \& Lauder 2016, Zhu et al. 2019). 
Wainwright \& Lauder (2016) hacen referencia a estas estructuras en el pez sol Lepomis macrochirus, que, si bien no está relacionada con la especie en estudio, ayuda a entender la presencia de ambos tipos de escamas y la función que presentan cada una de sus estructuras. Estos autores proponen algunas hipótesis acerca de la función mecánica de sólidos que tiene las escamas de los peces. Por ejemplo, la curvatura de las escamas puede aumentar la rigidez a la flexión permitiéndole aumentar la resistencia en el plano anterior-posterior, hecho que pudiera ser importante para reducir las lesiones o deformaciones locales durante la natación y en el caso de eventos de escape. Otro aspecto mencionado es que las escamas pueden almacenar energía elástica durante una locomoción ondulatoria estándar o lateral con altas maniobras de flexión, tales como respuestas de huida. Así entonces la energía elástica almacenada sería devuelta al pez para acelerar la flexión lateral del cuerpo, en la dirección opuesta y la curvatura de la escama afectaría la cantidad de energía almacenada.

En el caso de los circuli, incluyendo otras características de microrrugosidad de la superficie de la escama, pueden funcionar para limitar las tensiones de tracción, minimizándola durante la flexión de las escamas (Yang et al. 2013, Wainwright \& Lauder 2016). Respecto de los ctenii, Wainwright \& Lauder (2016) consideran que estas estructuras, se encuentran entrelazadas en el borde posterior de las escamas y pueden funcionar como regiones de flexibilidad, ya que no son tan rígidos como el cuerpo de la escama que presenta tejido sólido osificado. Esta hipótesis depende de la cinemática de las escamas durante la natación y asume que la parte posterior de una escama tocará la escama posterior a ella, creando una fuerza de flexión.

En el caso de los radii, Wainwright \& Lauder (2016) citando el trabajo de Taylor (1916), indican que estas estructuras permiten una mayor flexibilidad de la escama, reduciendo así la flexión del cuerpo y el costo locomotor del transporte debido a que son estructuras huecas en la capa osificada en las escamas.

En el área de la mecánica de fluidos, la morfología de las escamas también tiene una importante función (Liao 2007, Vernerey \& Barthelat 2010, 2014; Wainwright \& Lauder, 2016). En el caso de los ctenos, éstos controlan el flujo de agua disminuyendo la resistencia de fricción y con ello aumentan la energía de turbulencia en la capa del borde de la escama. Dicha turbulencia es definida como el movimiento heterogéneo tridimensional, temporal y espacialmente dependiente de las rápidas fluctuaciones de la velocidad del flujo que resultan de la superposición de flujos caóticos o críticos de múltiples fuerzas y tamaños (Kirkbride 1993, Silva et al. 2012). Por su parte, aquellos ctenos presentes hacia el pedúnculo crean un aumento de la turbulencia que es útil para controlar su intensidad cercana al cuerpo. Se ha observado que el aumento de la cobertura de ctenii hacia la aleta caudal podría indicar el uso de un sistema de turbuladores, que retardarían la separación de la capa límite como cambios de gradiente de presión del fluido en la parte posterior del cuerpo en los peces (Wainwright \& Lauder 2016).

Al no existir referencias respecto de las escamas del tronco de $D$. eleginoides, con las que se puedan cotejar los resultados, exceptuando las de Voronina \& Hughes (2017), se infiere que la descripción de Wainwright \& Lauder (2016) permite asumir que la funcionalidad de las escamas corporales es extrapolable a los ejemplares aquí reportados. Del mismo modo, la morfología externa de las escamas corporales y de ambas líneas laterales descritas en el presente estudio, aportan elementos morfológicos básicos desconocidos para esta especie. No obstante a ello, para el caso particular de las líneas laterales, es necesario continuar con estudios histológicos que permitan determinar si ambas líneas laterales son equivalentes en su función mecanosensorial y si existe alguna conexión nerviosa entre ambas líneas de $D$. eleginoides.

\section{Agradecimientos}

Los autores agradecen al Dr. Luis Vargas Ch. (Instituto de Ciencias Marinas y Limnológicas, Universidad Austral de Chile) por su permanente apoyo en esta investigación. A Stefany Camelo y Benjamin Guzman por su colaboración durante el proceso obtención y procesamiento de las muestras de escamas. Estos son resultados parciales del proyecto DID S-2017-25, financiado por la Dirección de Investigación de la Universidad Austral de Chile.

\section{LiTERATURA CITADA}

Alkaladi A, ASA Harabawy \& IAA Mekkawy. 2013. Scale characteristics of two fish species, Acanthopagrus bifasciatus (Forsskål, 1775) and Rhabdosargus sarba (Forsskål, 1775) from the Red Sea at Jeddah, Saudi Arabia. Pakistan Journal of Biological Sciences 16(8): 362-371.

Ansari S, S Chavan \& S Padghane. 2016. Morphology of scales in three teleost species from Godavari river basin in parts of Maharashtra, India. International Journal of Zoology Studies 1(6): 18-22.

Barbin C \& JC Humphrey. 2009. Drag force acting on a neuromast in the fish lateral line trunk canal. I. Numerical modelling of external-internal flow coupling. Journal of the Royal Society Interface 6: 627-640.

Batts BS. 1964. Lepidology of the adult Pleuronectiform fishes of Puget Sound, Washington. Copeia 1964(4): 666-673.

Burdak VD. 1986. Morphologie fonctionnelle du tegument ecailleux des poissons. Cybium 10: 1-128.

Ghysen A, K Schuster, D Coves, F de la Gandara, N Papandroulakis \& A Ortega. 2010. Development of the posterior lateral line system in Thunnus thynnus, the Atlantic blue-fin tuna, and in its close relative Sarda sarda. The International Journal of Developmental Biology 54: 1317-1322. 
Gompel N, N Cubedo, C Thisse, B Thisse, C DamblyChaudiere \& A Ghysen. 2001. Pattern formation in the lateral line of zebrafish. Mechanisms of Development 105: 69-77.

Ishida Y, R Asaoka, M Nakae \& K Sasaki. 2014. The trunk lateral line system and its innervation in Mugil cephalus (Mugilidae: Mugiliformes). Ichthyological Research 62(3): 253-255.

Jawad LA. 2005. Comparative scale morphology and squamation patterns in triplefins (Pisces: Teleostei: Perciformes: Tripterygiidae). Tuhinga 16: 137-167.

Kasumyan AO. 2003. The lateral line in fish: Structure, function, and role in behavior. Journal of Ichthyology 43 (Suppl. 2): 175-213.

Kirkbride AD. 1993. Observations of the influence of bed roughness on turbulence structure in depth limited flows over gravel beds. In: Clifford NJ, JR French \& J Hardisty (eds). Turbulence: perspectives on flow and sediment transport, pp. 185-196. John Wiley \& Sons, Chichester.

Liao J. 2007. A review of fish swimming mechanics and behaviour in altered flows. Philosophical Transactions of the Royal Society B 362: 1973-1993.

Lippitisch E. 1990. Scale morphology and squamation patterns in cichlids (Teleostei, Perciformes): A comparative study. Journal of Fish Biology 37: 265-291.

Lloris D \& J Rucabado. 1991. Ictiofauna del Canal Beagle (Tierra del Fuego). Aspectos ecológicos y análisis biogeográfico. Publicaciones Especiales, Instituto Español de Oceanografía 8: 1-182.

Modgans J, J Engelmann, W Hanke \& S Kröther. 2003. The fish lateral line: how to detect hydrodynamic stimuli. In: Barth FG, JAC Humphrey \& TW Secomb (eds). Sensors and sensing in biology and engineering, pp. 173-185. Springer-Verlag, Wien.

Ristroph L, JC Liao \& J Zhang. 2015. Lateral line layout correlates with the differential hydrodynamic pressure on swimming fish. Physical Review Letters 114: 018102. $<$ https://doi.org/10.1103/PhysRevLett.114.018102>

Roberts CD. 1993. Comparative morphology of spined scales and their phylogenetic significance in the Teleostei. Bulletin of Marine Science 52(1): 60-113.

Silva AT, C Katopodis, JM Santos, MT Ferreira \& AN Pinheiro. 2012. Cyprinid swimming behaviour in response to turbulent flow. Ecological Engineering 44: 314-328.

Taylor HF. 1916. The structure and growth of the scales of the squeteague and the pigfish as indicative of life history. Bulletin of the United States Bureau of Fisheries 34: 285-330.

Teimori A. 2016. Scanning electron microscopy of scale and body morphology as taxonomic characteristics of two closely related cyprinid species of genus Capoeta Valenciennes, 1842 in southern Iran. Current Science 111(7): 1214-1219.
Vernerey FJ \& F Barthelat. 2010. On the mechanics of fish scale structures. International Journal of Solids and Structures 47(17): 2268-2275.

Vernerey FJ \& F Barthelat. 2014. Skin and scales of teleost fish: Simple structure but high performance and multiple functions. Journal of the Mechanics and Physics of Solids 68: 66-76.

Vogel D \& H Bleckmann. 2000. Behavioral discrimination of water motions caused by moving objects. Journal of Comparative Physiology A 186(12): 1107-1117.

Voronina E. 2007. Diversity of the structure of lateral line scales in Pleuronectiformes. Journal of Ichthyology 47(3): 207-216.

Voronina E \& DR Hughes. 2011. Types and development pathways of lateral line scales in some teleost species. Acta Zoologica 94: 154-166.

Voronina E \& DR Hughes. 2017. Lateral line scale types and review of their taxonomic distribution. Acta Zoologica 1-17.

Wada H, S Hamaguchi \& M Sakaizumi. 2008. Development of diverse lateral line patterns on the teleost caudal fin. Developmental Dynamics 237(10): 2889-2902.

Wainwright DK \& VG Lauder. 2016. Three-dimensional analysis of scale morphology in bluegill sunfish, Lepomis macrochirus. Zoology 119: 182-195.

Webb J. 1989. Gross morphology and evolution of the mechanoreceptive lateral line system in teleost fishes. Brain, Behavior and Evolution 33: 34-53.

Webb J. 2014. Morphological diversity, development and evolution of the mechanosensory. In: Coombs S, H Bleckmann, RR Fay \& AN Popper (eds). Lateral line system 48: 17-72. Springer. Handbook of Auditory Research, New York.

Webb J \& JB Ramsay. 2017. New interpretation of the 3-D configuration of lateral line scales and the lateral line canal contained within them. Copeia 105(2): 339-347.

Wonsettler A \& J Webb. 1997. Morphology and development of the multiple lateral line canals on the trunk in two species of Hexagrammos (Scorpaeniformes, Hexagrammidae). Journal of Morphology 233(3): 195-214.

Yang W, IH Chen, B Gludovatz, EA Zimmermann, R Ritchie \& MA Meyers. 2013. Natural flexible dermal armor. Advanced Materials Interfaces 25: 31-48.

Zhu D, C Fuentes-Ortega, R Motamedi, L Szewciw, F Vernerey \& F Barthelat. 2012. Structure and mechanical performance of a "modern" fish scale. Advanced Engineering Materials 14(4): B185-B194.

Zhu D, Ch Zhang, PL Liu \& A Jawad. 2019. Comparison of the morphology, structures and mechanical properties of teleost fish scales collected from New Zealand. Journal of Bionic Engineering 16: 328-336. 